\title{
Vingt-cinq ans d'actions concertées françaises sur les écoulements diphasiques
}

\author{
Twenty five years of French cooperative research on two phase flows
}

\author{
par Jean-Marc Delhaye \\ Directeur de Recherches au CEA \\ Professeur à l'Ecole Centrale Paris \\ Directeur du GREDIC (GDR 1205 du CNRS)
}

Gas-liquid or liquid-vapor two-phase flows are encountered in many industrial systems or processes, such as nuclear reactors, rocket cryogenic engines, chemical contactors, oil wells, pipe-lines, evaporators or condensers. Offers for research and development by the French laboratories are exemplified in this paper. Cooperative research programs have been carried out in France for more than twenty-five years and have led to a network of French laboratories which are now internationally recognized. They have also allowed technology ressources to be transferred from basic research to industrial applications.

\section{1 L'ORIGINE DES ACTIONS CONCERTÉES}

Au début des années 1970, l'effort principal de recherche et de développement sur les écoulements diphasiques se situait essentiellement dans le domaine nucléaire autour des écoulements eau-vapeur pouvant être rencontrés dans les réacteurs électrogènes, les réacteurs de propulsion navale et les réacteurs de recherche. Cependant le nombre croissant de problèmes complexes soulevés, tant du point de vue théorique qu'appliqué, a rapidement incité les responsables du CEA et d'EDF à convaincre le CNRS de lancer en 1976 une Action Thématique Programmée sur les écoulements diphasiques. Parallèlement, la Délégation Générale à la Recherche Scientifique et Technique (DGRST), devant les nombreuses applications industrielles où intervenait la complexité des écoulements diphasiques, soutenait depuis 1972 une action concertée importante dans le même domaine. Ces deux actions ont permis, non seulement d'acquérir des résultats scientifiques et techniques pertinents mais surtout de créer des équipes de recherche françaises dont les compétences sont actuellement largement reconnues sur le plan international comme en témoigne en particulier l'organisation par le Professeur Jean Bataille de la Third International Conference on Multiphase Flow (ICMF'98) qui a eu lieu à Lyon du 8 au 12 juin 1998.

\section{LE CONTRAT DE PROGRAMME SUR LES ÉCOULEMENTS DIPHASIQUES INDUS- TRIELS NON NUCLÉAIRES (1986-1990)}

\subsection{L'évaluation des besoins industriels}

Dès 1979, le Comité Mécanique de la DGRST avait financé une enquête approfondie sur les blocages scientifiques et techniques liés à la mécanique des fluides dans cinq secteurs industriels : traitement des eaux, papeterie, agroalimentaire, transmissions et commandes hydrauliques et génie chimique. Cette enquête, confiée à la Société Bertin et suivie par un groupe d'experts du Comité Mécanique a permis d'identifier huit grands axes de recherche prioritaires en mécanique des fluides. Parmi ceux-ci, la filtration et la rhéologie, qui furent l'objet de contrats de programmes lancés respectivement en 1982 et 1984 , et les écoulements diphasiques qui généraient de nombreux problèmes auxquels se trouvaient confrontés en particulier des secteurs industriels très concernés comme le traitement des eaux, le génie chimique et le secteur énergétique (production et utilisation rationnelle de l'énergie).

\subsection{La mise en place du contrat de programme}

A l'initiative de la Société Bertin et du CEA, une proposition de contrat de programme sur les écoulements diphasiques industriels non nucléaires a été élaborée en 1984 et soumise à divers organismes susceptibles de financer les travaux. Deux objectifs étaient clairement exprimés :

1. transférer des connaissances acquises dans le secteur nucléaire vers le secteur mécanique-énergétique non nucléaire,

2. acquérir des connaissances nouvelles répondant aux préoccupations de ce dernier secteur.

Le contrat de programme a réuni en tant que partenaires, le CEA/Grenoble, la Société Bertin et les six laboratoires suivants du CNRS :

- le Laboratoire de Mécanique des Fluides et d'Acoustique, Lyon (ECL),

- le Laboratoire d'Energétique et de Mécanique Théorique et Appliquée, Nancy (LEMTA),

- l'Institut de Mécanique des Fluides de Toulouse (IMFT), 
- l'Institut de Mécanique de Grenoble (IMG),

- le Laboratoire de Phénomènes de Transport dans les Milieux en Réaction, Rouen (LESP, CORIA),

- le Laboratoire de Physique et Mécanique des Milieux Hétérogènes, Paris (ESPCI).

Le financement s'est étendu sur cinq années budgétaires (1985 à 1989) et a été assuré par le MRT, l'AFME, le CNRS, le CEA, EDF et la Société Bertin.

Les travaux ont été regroupés en six thèmes :

- conception et validité des modèles d'écoulements diphasiques en conduite (CEA/G),

- instabilités interfaciales (CEA/G, CORIA, Bertin),

- séparation des phases dans les singularités (CEA/G,

IMFT, LEMTA),

- écoulements dispersés multidimensionnels (ECL, IMFT, IMG, LESP, Bertin),

- transition entre écoulements à contre-courant et écoulements cocourants (CEA/G, LEMTA),

- techniques de mesure (CEA/G, IMG, ESPCI).

La gestion scientifique et financière du contrat de programme a été réalisée par l'intermédiaire d'un groupement, le GREDIC (Groupement pour la Recherche sur les Ecoulements Diphasiques en Conduite) qui a associé de 1985 à 1987 le CEA et le CNRS et à partir de 1987 le CEA, le CNRS, EDF et la Société Bertin.

\subsection{Bilan du contrat de programme}

Un colloque bilan s'est tenu au MRT en octobre $1990 \mathrm{au}$ cours duquel ont été présentées devant un parterre d'industriels les conclusions scientifiques et techniques du contrat de programme. Au cours de ce colloque, une session spéciale a été consacrée au savoir-faire français en matière de techniques de mesure utilisables dans les écoulements diphasiques. Cette session était placée sous le co-patronnage du MRT et de la Société Hydrotechnique de France dont la section Ecoulements Polyphasiques avait suscité une enquête sur les besoins industriels et sur les compétences des laboratoires français dans le domaine de l'instrumentation pour les écoulements diphasiques.

Les acquis scientifiques et techniques de ce contrat de programme sont décrits dans la référence [1]. Parmi les résultats significatifs, on notera en particulier :

- l'importance de la qualification des modèles d'écoulements diphasiques en conduite non seulement sur les caractéristiques globales de l'écoulement (pertes de pression, taux de vide) mais aussi sur les caractéristiques des phénomènes de propagation d'onde (célérité, amplification et amortissement),

- la mise en évidence de la structure des écoulements diphasiques dans les tuyauteries d'admission des moteurs à allumage commandé,

- la modélisation complète d'un atomiseur mécanique à swirl,

- la compréhension de la structure des écoulements dans les singularités telles que les élargissements brusques et les jonctions en té,

- la mise au point de modélisations fines des écoulements dispersés à bulles ou à gouttes tenant compte de la turbulence de la phase porteuse et des interactions entre cette dernière et les gouttes ou les bulles,

- la création d'une banque de données locales sur les écoulements dispersés dans des situations typiques telles qu'élargissements brusques, couches limites, jets, jonctions en té,

- la mise en cuvre de codes de calcul d'écoulements dispersés à bulles ou à gouttes dans des géométries complexes,

- la compréhension des mécanismes hydrodynamiques conduisant à la transition entre l'écoulement à contre-courant et l'écoulement cocourant dans le cas d'un film liquide ruisselant sur une paroi verticale et soumis à un écoulement de gaz à contre-courant,

- la mise au point et la qualification d'une technique de mesure permettant la détermination simultanée du taux de vide, de l'aire interfaciale et de la taille des bulles dans une colonne à bulles,

- la mise au point et la qualification d'un vélocimètre à réseau permettant la mesure de la vitesse des particules dans un écoulement,

- la démonstration des possibilités d'emploi de la résonance magnétique nucléaire en débitmétrie diphasique.

\section{III $\square$ LE GREDIC, GDR 1027 DU CNRS (1992-1995)}

A l'issue du contrat de programme précédent et après de nombreux contacts avec l'industrie, il est apparu que de nombreux problèmes nécessitaient la modélisation d'écoulements diphasiques multidimensionnels et qu'un effort soutenu devait être poursuivi pour le développement de techniques de mesure en écoulement diphasique.

C'est pourquoi, à la demande de la Commission de suivi du contrat de programme et du Comité directeur du GREDIC, il a été proposé au CNRS la création d'un groupement de recherche (GDR) intitulé Ecoulements diphasiques : modélisation multidimensionnelle et techniques de mesure.

\subsection{Les partenaires}

Créé le $1^{\text {er }}$ janvier 1992, le groupement de recherche 1027 du CNRS a gardé l'appellation GREDIC et a rassemblé de façon très étroite les équipes suivantes du CNRS, du CEA, d'EDF et de la Société Bertin :

CNRS (Département Sciences Pour l'Ingénieur)

- Laboratoire de Mécanique des Fluides et d'Acoustique, Lyon (ECL),

- Institut de Mécanique des Fluides de Toulouse (IMFT),

- Laboratoire des Phénomènes de Transport dans les Milieux en Réaction, Rouen (LESP, CORIA),

- Laboratoire d'Energétique et de Mécanique Théorique et Appliquée, Nancy (LEMTA),

- Laboratoire des Ecoulements Géophysiques et Industriels, Grenoble (LEGI),

- Laboratoire de Physique et de Mécanique des Milieux Hétérogènes, Paris (ESPCI).

$E D F$ (Direction des Etudes et Recherches)

- Département Transferts Thermiques et Aérodynamique (TTA),

- Laboratoire National d'Hydraulique (LNH).

CEA (Direction des Réacteurs Nucléaires)

- Service de Thermohydraulique pour les Applications Industrielles, Grenoble (CEA/G)

SOCIÉTÉ BERTIN ET CIE

- Division Mécanique des Fluides 


\section{- 3.2 Les thèmes de recherche}

De 1992 à 1995 les actions du GREDIC ont été regroupées en deux thèmes : la modélisation multidimensionnelle des écoulements dispersés et les techniques de mesure adaptées aux écoulements diphasiques gaz-liquide.

Le thème Modélisation multidimensionnelle a comporté sept actions :

(1) Transferts pariétaux et interfaciaux dans les écoulements à bulles ; application au mélangeage diphasique,

(2) Ecoulements turbulents multidimensionnels à bulles,

(3) Modélisation des écoulements dispersés denses,

(4) Modélisation des pulvérisateurs,

(5) Ecoulements diphasiques en rotation,

(6) Comportement numérique des modèles multidimensionnels d'écoulement diphasique,

(7) Modélisation des écoulements dispersés multidimensionnels.

Le thème Techniques de mesure a regroupé cinq actions :

(8) Techniques tomographiques en écoulement diphasique,

(9) Diffusion de la lumière par les écoulements diphasiques,

(10) Caractérisation d'une dispersion liquide-liquide vis-à-vis de la coalescence,

(11) Qualification d'une méthode utilisant la RMN en vue de déterminer les fractions volumiques et les débits des phases dans un écoulement diphasique,

(12) Développement de techniques de mesure locale pour les écoulements diphasiques.

\subsection{Le fonctionnement du GREDIC}

Au cours de ces quatre années, les partenaires du GREDIC se sont rencontrés fréquemment soit dans le cadre de réunions propres au GREDIC, soit au cours de manifestations scientifiques diverses (congrès français et internationaux, jurys de thèse, comités scientifiques de laboratoires, etc.). Ils ont participé également de façon active à deux autres GDR du CNRS (microgravité et mécanique des fluides numérique).

Des réunions plénières annuelles ont été organisées à Grenoble (6-7 avril 1993) et Toulouse (30-31 avril 1994). Par ailleurs une réunion de présentation du GREDIC a eu lieu à Grenoble le 27 janvier 1995 et a rassemblé quarante-cinq participants dont la moitié d'industriels. Enfin un colloquebilan sur les avancées scientifiques du GREDIC s'est déroulé à Paris le 23 juin 1995. Les communications qui y ont été présentées ont fait l'objet d'un numéro thématique de La Houille Blanche [2]. Reconnaissant l'impact du GREDIC dans le domaine de la mécanique des fluides, la Société Hydrotechnique de France a apporté son parrainage aux deux manifestations précédentes.

Au cours de ses quatre années de fonctionnement, 1992 à 1995, le GREDIC a été soutenu financièrement par le CNRS, le CEA, EDF, le MENESRIP et la Société Bertin.

\subsection{Les retombées}

La production scientifique du GREDIC sur les années 1992-1995 a été particulièrement féconde comme le montrent les articles publiés en [2] ainsi que le nombre des thèses soutenues et en cours dans les laboratoires partenaires du GREDIC. On notera également l'importance des contrats obtenus par les partenaires avec différents organismes ou industriels, et des relations internationales par le biais de réseaux et de participations à des manifestations.
Les partenaires du GREDIC ont participé de façon très active à deux conférences internationales :

- le Third International Workshop on Two-Phase Flow Fundamentals, Londres, 1992,

- la Second International Conference on Multiphase Flow, Kyoto, 1995.

$\mathrm{La}$ reconnaissance internationale des travaux français sur les écoulements diphasiques ainsi que le dynamisme des équipes concernées ont d'ailleurs permis à la France d'être choisie comme le pays organisateur de la Third International Conference on Multiphase Flow (ICMF'98). Celle-ci a eu lieu à Lyon du 8 au 12 juin 1998 sous la présidence du professeur Jean Bataille.

\section{IV — LE GREDIC, GDR 1205 DU CNRS (1996-1999)}

Une collaboration étroite et féconde entre les partenaires du GREDIC, des contacts suivis et actifs avec les laboratoires étrangers, l'émergence de problèmes de base venant de problèmes industriels ont conduit le CNRS à prolonger les activités du GDR 1027. Le nouveau groupement, le GDR 1205 , Instabilités et turbulence dans les écoulements diphasiques, a gardé l'appellation GREDIC mais se distingue du précédent par :

1. l'apparition d'actions nouvelles concernant la dynamique des interfaces,

2. l'arrêt des actions sur les techniques de mesure pour lesquelles le relais peut être pris par d'autres instances,

3. l'apparition de deux équipes nouvelles : le LIMSI (Orsay) et le Laboratoire de Modélisation en Mécanique (Paris).

\section{Les thèmes de recherche}

L'ensemble du programme comporte 20 actions regroupées en deux thèmes : la dynamique des interfaces et les écoulements dispersés. L'ensemble constitue une contribution à la compréhension des phénomènes d'instabilité et de turbulence dans les écoulements diphasiques. Les 20 actions en cours sont répertoriées dans le tableau 1 où figurent le titre, le laboratoire et le responsable scientifique pour chaque action. Le document [3] décrit de façon détaillée chacune des 20 actions. Les activités du GREDIC du 1.01 .96 au 30.06.97 sont décrites par action dans le document [4].

Bien que le thème Techniques de mesure ne figure plus explicitement dans le programme actuel du GREDIC, les partenaires du GREDIC ont participé de façon très active à la réunion des spécialistes en techniques de mesure pour les écoulements diphasiques qui a été organisée par l'OCDE à Santa Barbara du 17 au 20 mars 1997. Six communications invitées de partenaires du GREDIC y ont été présentées.

\section{L LE GROUPEMENT POUR LA RECHERCHE SUR LES ÉCHANGEURS THERMIQUES (GRETh)}

L'amélioration des technologies industrielles vers une sobriété et une propreté accrues constitue un enjeu majeur pour notre économie. Les échangeurs de chaleur et autres équipements thermiques comme les chaudières, les fours, les incinérateurs, les condenseurs, etc. sont autant d'éléments essentiels de toute politique de maîtrise de l'énergie et de protection de l'environnement. Ces équipements se retrouvent aussi bien dans les procédés industriels (chimie, pétrochimie, sidérurgie, agro-alimentaire,...), que dans les secteurs 
des transports (automobile, aéronautique) et de l'habitat (climatisation, chauffage,...).

Etant donné la diversité et la dissémination du tissu industriel concerné, il est apparu important d'organiser la recherche à caractère finalisé et de favoriser l'innovation dans les entreprises, particulièrement les PME-PMI en leur transférant les connaissances acquises. C'est pour répondre à ces objectifs que le CEA et l'Ademe (Agence de l'environnement et de la maîtrise de l'énergie) ont créé en 1984 le Groupement pour la recherche sur les échangeurs thermiques, le GRETh, qui a pour vocation d'apporter son soutien aux industriels dans les domaines de la thermique industrielle. Il s'adresse aux bureaux d'ingéniérie, aux utilisateurs et prescripteurs, grandes et petites entreprises, aux fabricants de matériels thermiques..., et regroupe actuellement une centaine d'adhérents. Le GRETh est situé sur le centre de Grenoble du CEA.

\section{Le GRETh : centre de ressources technologiques}

Les trois principaux objectifs du GRETh sont les suivants : 1. améliorer les connaissances de base sur les équipements de transfert thermiques,

2. mettre au point de nouvelles conceptions de produits, plus performants et moins onéreux,

3. développer des programmes de simulation numérique et des banques de données.

Ce groupement de recherche se préoccupe plus particulièrement des transferts thermiques en écoulement diphasique avec évaporation et condensation, des échanges de chaleur à haute température, des applications performantes de l'électricité et des technologies propres et économes (équipements de dépollution de l'air, traitement des composés organiques volatils, valorisation et traitement de déchets, utilisation des fluides frigoriferes à faible impact sur l'environnement,...).

En plus de sa propre capacité de recherche, le GRETh utilise les compétences des chercheurs du pôle thermohydraulique de Grenoble : Département de thermohydraulique et de physique du CEA, Université Joseph Fourier, Institut National Polytechnique de Grenoble, avec lesquels il effectue les recherches de base qui ne peuvent être prises en charge par les industriels, en particulier par les PME-PMI. Il s'appuie également sur le CNRS (programme ECODEV) pour conduire des recherches plus amont.

De grands organismes publics (CEA, EDF, Ademe, Union européenne) alimentent $70 \%$ du budget du GRETh, le reste étant apporté par les industriels adhérents.

Le GRETh associe en outre à ses études des centres techniques industriels tels que le CETIAT (Centre technique des industries aérauliques et thermiques) et le CETIM (Centre technique des industries de la mécanique) qui sont, avec le CEA et l'Ademe, membres fondateurs du Club GRETh, association loi 1901 regroupant les industriels adhérents du GRETh.

\section{VI — CONCLUSION}

Les actions sur les écoulements diphasiques menées depuis plus de vingt-cinq ans ont permis à la France de disposer d'équipes particulièrement compétentes pour entreprendre des recherches de base finalisées sur les écoulements diphasiques et en faire diffuser les résultats vers les demandeurs industriels. Cet effort doit être poursuivi pour contribuer à rendre nos entreprises toujours plus innovantes.

\section{Références}

[1] Delhaye, J.M., Les actions concertées sur les écoulements diphasiques : motivations, bilans et perspectives, Colloque Bilan 1991, Recherches soutenues par le Ministère de la recherche et de la technologie dans le secteur de la mécanique, Société Française des Mécaniciens (Courbevoie), 1991.

[2] Les écoulements diphasiques (modélisation et technique de mesure), La Houille Blanche, No 1/2, pp 46-134, 1996.

[3] DelhaYe, J.M., Instabilités et turbulence dans les écoulements diphasiques, Demande de renouvellement du Groupement de Recherche 1027 (GREDIC) auprès du CNRS, 1995

[4] Del.hAYE, J.M., Instabilités et turbulence dans les écoulements diphasiques, Rapport au 30.06.97 du GDR 1205 du CNRS, 1997.

\begin{tabular}{|c|c|c|c|}
\hline \multicolumn{4}{|c|}{ Tableau 1 : Les actions en cours au GREDIC } \\
\hline \multicolumn{2}{|r|}{ DYNAMIQUE DES INTERFACES } & \multicolumn{2}{|r|}{ ECOULEMENTS DISPERSÉS } \\
\hline 1 & $\begin{array}{l}\text { Mécanismes de pulvérisation. Etude expérimentale et modélisation. - } \\
\text { CORIA, Rouen. M. LEDOUX }\end{array}$ & 11 & $\begin{array}{l}\text { Etude des écoulements à bulles pilotés par la gravité. - LMFA, Lyon } \\
\text { M. LANCE }\end{array}$ \\
\hline 2 & $\begin{array}{l}\text { Déstabilisation de films liquides par des effets gravitaires ou centrifuges. } \\
\text { - LPMMH, Paris. L. LIMAT }\end{array}$ & 12 & $\begin{array}{l}\text { Macrosimulation des écoulements gaz-liquide à bulles - LMFA, } \\
\text { Lyon M. LANCE. }\end{array}$ \\
\hline 3 & $\begin{array}{l}\text { Instabilités de films liquides cisaillés par un gaz. - IMFT, Toulouse } \\
\text { F. CHARRU }\end{array}$ & 13 & $\begin{array}{l}\text { Simulation des grandes échelles des écoulements turbulents dipha- } \\
\text { siques dispersés. - IMFT, Toulouse. J. MAGNAUDET }\end{array}$ \\
\hline 4 & $\begin{array}{l}\text { Stabilité des films liquides en conduite avec interaction à l'interface. - } \\
\text { LEMTA, Nancy. B. IZRAR }\end{array}$ & 14 & $\begin{array}{l}\text { Développement et validation d'une modélisation eulerienne des écou- } \\
\text { lements turbulents diphasiques à inclusions dispersées. - LNH, Cha- }\end{array}$ \\
\hline \multirow{2}{*}{5} & \multirow{2}{*}{$\begin{array}{l}\text { Contrôle par champ magnétique de la stabilité d'interfaces en repère tour- } \\
\text { nant. - LEMTA, Nancy, M. SOUHAR }\end{array}$} & & tou O. SIMONIN \\
\hline & & \multirow[t]{2}{*}{15} & \multirow{2}{*}{$\begin{array}{l}\text { Dynamique des nuages de particules. - LESP, Rouen. A.BERLE- } \\
\text { MONT }\end{array}$} \\
\hline \multirow[t]{2}{*}{6} & \multirow{2}{*}{$\begin{array}{l}\text { Etude de l'instabilité d'une interface liquide-vapeur horizontale pour la } \\
\text { maîtrise de l'ébullition en film. - LIMSI, Orsay, P. LE QUÉRÉ }\end{array}$} & & \\
\hline & & \multirow[t]{2}{*}{16} & \multirow{2}{*}{$\begin{array}{l}\text { Pseudo-turbulence en écoulements dispersés. - LEGI, Grenoble. } \\
\text { A. CARTELLIER }\end{array}$} \\
\hline \multirow[t]{2}{*}{7} & \multirow{2}{*}{$\begin{array}{l}\text { Application de la description continue d'une interface liquide - vapeur à } \\
\text { la dynamique de systèmes diphasiques en présence de changement de } \\
\text { phase. - CEA, Grenoble, O. LEBAIGUE }\end{array}$} & & \\
\hline & & 17 & $\begin{array}{l}\text { Pseudo-turbulence dans les milieux diphasiques dispersés. - LMM, } \\
\text { Paris. D. LHUILLIER }\end{array}$ \\
\hline \multirow[t]{2}{*}{8} & \multirow{2}{*}{$\begin{array}{l}\text { Caractérisation des changements de configuration des écoulements dipha- } \\
\text { siques gaz-liquide : développement et mise en cuvre de méthodes avan- } \\
\text { cées de traitement du signal. - CEA, Grenoble, E. HERVIEU }\end{array}$} & 18 & $\begin{array}{l}\text { Modélisation fine des transferts de masse et de chaleur dans les écou- } \\
\text { lements eau-vapeur en ébullition. - TTA, Chatou. E. BRIERE }\end{array}$ \\
\hline & & \multirow[t]{2}{*}{19} & \multirow{2}{*}{$\begin{array}{l}\text { Modélisation multidimensionnelle des milieux diphasiques denses. - } \\
\text { Bertin, Plaisir. E. ZNATY }\end{array}$} \\
\hline \multirow[t]{2}{*}{9} & \multirow{2}{*}{$\begin{array}{l}\text { Caractérisation de systèmes dynamiques complexes.- LESP, Rouen } \\
\text { G. GOUESBET }\end{array}$} & & \\
\hline & & \multirow[t]{2}{*}{20} & \multirow{2}{*}{$\begin{array}{l}\text { Instabilités et turbulence dans les écoulements diphasiques. - IMFT, } \\
\text { Toulouse. CH. SUZANNE }\end{array}$} \\
\hline 10 & $\begin{array}{l}\text { Structure de l'écoulement intermittent vertical ascendant. - IMFT, Tou- } \\
\text { louse, J. FABRE }\end{array}$ & & \\
\hline
\end{tabular}

